\title{
Um Inquérito Sobre a Opinião, Conhecimentos e Necessidades de Formação dos Médicos Relativamente aos Alimentos Transgénicos
}

\section{A Survey Study Assessing Opinions, Knowledge and Training Needs of Physicians Concerning the Field of Transgenic Foods}

\author{
Isabella VIEIRA ${ }^{1}$, Teresa BRANDÃO ${ }^{1}$, Elisabete PINTO ${ }^{1,2}$, Margarida SILVA $^{1}$ \\ Acta Med Port 2020 Apr;33(4):252-260 - https://doi.org/10.20344/amp.11946
}

\section{RESUMO}

Introdução: Há alimentos transgénicos (modificados geneticamente) a serem cultivados, comercializados e consumidos em Portugal. É importante que os médicos se mantenham ao corrente quanto aos impactos de tais alimentos na saúde. Este trabalho propõe-se identificar a opinião, conhecimento e necessidades de formação dos médicos face aos alimentos transgénicos.

Material e Métodos: Foi aplicado um inquérito a 278 médicos.

Resultados: Em relação à opinião, 85,8\% apresentam uma posição neutra, $12,5 \%$ negativa e uma minoria $(1,7 \%)$ positiva. No entanto, $79,8 \%$ têm um nível de conhecimento baixo, $17,7 \%$ médio e $2,5 \%$ alto. De referir que $91,4 \%$ consideram útil existir algum tipo de formação durante a educação universitária e $65,1 \%$ destacaram ser muito útil ao longo da vida profissional a existência de formação continuada sobre esta temática. Emergiram dois perfis de médicos: um grupo (83\%), interessado em saber mais sobre alimentos transgénicos e outro grupo (17\%), desinteressado.

Discussão: Os médicos são considerados fontes de referência fiável a quem a população opta por recorrer para obter informação sobre saúde, o que sugere a necessidade de se manter um conhecimento suficiente e atualizado sobre este tema.

Conclusão: Este estudo é pioneiro no que se refere ao posicionamento de médicos portugueses relativamente a estes alimentos. Os resultados apontam para uma necessidade de formação específica.

Palavras-chave: Alimentos Geneticamente Modificados; Conhecimentos, Atitudes e Prática em Saúde; Médicos; Portugal

\section{ABSTRACT}

Introduction: Transgenic (genetically modified) foods are being grown, sold and eaten in Portugal. As such it is important that physicians stay up to date on any negative health consequences of such foods. This study aimed to identify their opinion, knowledge and training needs regarding transgenic foods.

Material and Methods: A total of 278 physicians responded to a survey

Results: In terms of opinion, $85.8 \%$ of physicians had a neutral position, $12.5 \%$ had a negative view and a minority (1.7\%) was positive. However, $79.8 \%$ had a low level of knowledge, $17.7 \%$ had an average level of knowledge and only $2.5 \%$ showed a high level of knowledge regarding the subject. It should be noted that $91.4 \%$ of physicians consider that it is useful to carry out some kind of training during their university education and $65.1 \%$ said that it would be very useful during the professional life to have continuing education concerning the issue. Most physicians (83\%) could be grouped together according to their interest in learning more. The other group $(17 \%)$ showed lack of interest.

Discussion: Physicians are considered reliable sources, to whom the general population can turn to in order to access valuable health information, which suggests the need to stay up-to-date regarding transgenic foods.

Conclusion: This is a pioneering study on Portuguese physicians and transgenic foods. Results point to a need for specific training concerning this issue.

Keywords: Food, Genetically Modified; Health Knowledge, Attitudes, Practice; Physicians; Portugal

\section{INTRODUÇÃO}

Com os avanços da engenharia genética e da aplicação das técnicas de manipulação e recombinação de genes à agricultura e alimentação surgem novas realidades, novas oportunidades e, potencialmente, novos perigos. ${ }^{1} \mathrm{~A}$ comercialização de alimentos transgénicos (AT), que atualmente aparecem em Portugal sobretudo em produtos com milho ou soja, é um dos resultados recentes destes avanços tecnológicos e tem gerado discussão relativamente às implicações na saúde e no meio ambiente, eventualmente associadas ao seu consumo. ${ }^{1,2} \mathrm{De}$ acordo com a Diretiva Europeia 2001/18, transgénicos são os organismos "cujo material genético tenha sido modificado de uma forma que não ocorre naturalmente por meio de cruzamentos e/ou de recombinação natural". ${ }^{3}$ Segundo as empresas agrobiotecnológicas que os desenvolvem (Monsanto, Bayer, Pioneer, etc), a modificação genética dos alimentos visa a melhoria da sua qualidade, o aumento da produção e o aumento da resistência a pragas e outras limitações agroambientais. ${ }^{4}$ Existe, no entanto, desconhecimento e insegurança por parte dos consumidores europeus sobre a sua introdução, temendo possíveis efeitos negativos na saúde e no ambiente, quer a curto, quer a longo prazo. ${ }^{4,5} \mathrm{~A}$ própria

1. Centro de Biotecnologia e Química Fina, Laboratório Associado. Escola Superior de Biotecnologia. Universidade Católica Portuguesa. Porto. Portugal.

2. Departamento de Nutrição e Saúde Pública. Instituto de Saúde Pública. Universidade do Porto. Porto. Portugal.

$\triangle$ Autor correspondente: Isabella Vieira. ifvieira@porto.ucp.pt

Recebido: 15 de fevereiro de 2019 - Aceite: 01 de agosto de 2019 | Copyright @ Ordem dos Médicos 2020 
legislação em vigor (Diretiva 2001/18)³ prevê a existência de um sistema de vigilância pós-comercialização uma vez que reconhece a impossibilidade de garantir o risco zero aquando da autorização do AT para introdução no mercado.

Em Portugal, e desde 2005, há cultivo, pouco extenso, de uma única variedade de milho geneticamente modificado (menos de $10 \%$ da área total dedicada ao milho), sendo que os AT presentes no mercado nacional são maioritariamente importados de países como os Estados Unidos e Brasil para utilização pela indústria das rações. ${ }^{6}$ Note-se que os AT (dezenas de variedades de milho e soja, o que corresponde a milhões de toneladas por ano) estão aprovados pela Comissão Europeia para consumo tanto animal como humano e para toda a União Europeia. ${ }^{7}$ Em Portugal o consumo direto de AT concentra-se atualmente nos óleos alimentares (soja) e alguns produtos importados de baixa expressão (milho). ${ }^{8,9}$

Os profissionais de saúde, médicos em particular, são considerados fontes de informação credível aos quais os pacientes recorrem para esclarecimento de temáticas diversas, incluindo o consumo de AT, ${ }^{10,11}$ devendo manter-se ao corrente quanto aos impactos de tais alimentos na saúde de quem os consome. A orientação prestada pelo profissional de saúde poderá ajudar os cidadãos, na tomada de posição individual em relação ao consumo de AT. Por conseguinte estes profissionais devem estar conscientes do seu papel determinante e capacitados a responder a questões desta temática, com um conhecimento profundo dos princípios científicos e sua relevância societal. ${ }^{12}$

O presente estudo teve como objetivo a caraterização da opinião, do conhecimento e da necessidade de formação da classe médica relativamente aos alimentos transgénicos.

\section{MATERIAL E MÉTODOS}

Este estudo foi baseado num inquérito no qual foram incluídas (i) sete questões de caracterização sociodemográfica dos respondentes, (ii) doze questões de opinião com foco em aspetos sociais, ambientais, nutricionais, consumo, saúde e informação aos consumidores, (iii) seis questões de conhecimento relacionadas com cultivo, venda e rotulagem de alimentos transgénicos em Portugal e vinte e cinco questões sobre perceção de necessidades de formação (questões fechadas de tipologia, temas e fontes de informação; e três perguntas fechadas dicotômicas e uma aberta descritas no item 3.4). Utilizaram-se questões fechadas em escalas qualitativas, tipo Likert com três níveis (discordo, nem discordo/nem concordo, concordo) ou de escolha múltipla com uma única possibilidade de resposta.

O questionário, criado na plataforma online do LimeSurvey (versão 1.91+), foi divulgado de março a julho de 2016 com apoio da Ordem dos Médicos, em encontros científicos e em redes sociais e profissionais. A disseminação abrangeu potencialmente uma população de 50927 médicos, número correspondente aos inscritos na Ordem, à altura do desenvolvimento do estudo. O estudo foi aprovado pelo Conselho Científico da Escola Superior de Biotecnolo- gia e pela Comissão de Ética da Católica Porto, Universidade Católica Portuguesa. Os dados foram exportados do LimeSurvey para Microsoft ${ }^{\circledR}$ Excel $^{\circledR}$ (Microsoft Corporation, versão 14.5.7) e analisados no programa IBM SPSS Statistics 23 for Windows ${ }^{\circledR}$ (SPSS Inc., Chicago, USA).

A opinião dos respondentes face aos AT foi classificada em negativa (discordo), neutra (nem discordo/nem concordo) e positiva (concordo) - com base no número de opiniões contra ou a favor. O conhecimento sobre os alimentos transgénicos foi classificado em baixo, médio e alto, conforme o número de respostas acertadas entre 0 2, 3 - 4 e 5 - 6, respetivamente. Respostas "não sei" foram contabilizadas como erradas.

Foram realizados testes de independência do qui-quadrado associados a uma análise de correspondência (ANA$\mathrm{COR})^{13}$ para investigar associações entre as questões e as características sociodemográficas dos médicos respondentes. Correlações baseadas no coeficiente de correlação de Spearman $\left(r_{\text {Spearman }}\right)$ foram também investigadas para variáveis ordinais, considerando-se um nível de significância de $7 \%{ }^{13}$

Realizou-se uma análise de correspondência múltipla $(\mathrm{ACM})^{14}$ visando a diminuição do número de variáveis e posterior análise de clusters de todas as questões, para obtenção de grupos de variáveis relacionadas entre si. Utilizou-se a distância Euclidiana quadrática como medida de similaridade entre os elementos a serem agrupados e o método de ligação de Ward como método aglomerativo. ${ }^{14} \mathrm{O}$ teste de independência do qui-quadrado foi também utilizado para obtenção das relações entre as questões e as categorias das respostas obtidas em cada cluster, assumindo-se um nível de significância de $5 \% .{ }^{14}$

\section{RESULTADOS}

A amostra final obtida foi de 278 médicos. Esta dimensão amostral supera o valor ideal de respondentes que seria de 248 elementos, com um erro amostral de $5 \%$ e nível de confiança de $95 \% .^{15}$

\section{Caracterização dos participantes}

Os resultados sobre a caracterização sociodemográfica dos participantes do estudo encontram-se na Tabela 1. O número de médicos respondentes foi de 278 , sendo $45,1 \%$ mulheres. Dois terços da amostra tinham idades compreendidas entre 31 e 60 anos e a maioria residia na região Norte $(36,3 \%)$ ou no Centro $(35,2 \%)$ do país. Constatou-se que uma proporção considerável dos profissionais possuía formação adicional para além da licenciatura: 17,6\% possuíam pós-graduação, 29,9\% mestrado e 6,5\% doutoramento. Relativamente ao local onde realizaram a sua formação académica, 33,5\% referiram a Faculdade de Medicina da Universidade de Coimbra, 32,7\% a Faculdade de Medicina da Universidade do Porto, 10,1\% a Faculdade de Medicina da Universidade de Lisboa e 8,6\% a Faculdade de Ciência Médicas da Universidade Nova de Lisboa. Em relação ao ano de conclusão do curso, 38,4\% concluíram entre 2005 e 2016. Perguntou-se, também, a 
frequência com que os profissionais contactavam com pacientes, sendo que $87,4 \%$ referiu fazê-lo diariamente.

\section{Caracterização da opinião dos médicos em relação aos alimentos transgénicos}

As opiniões dos médicos foram agregadas em função de três grandes conceitos: (i) aspetos sociais, ambientais e nutricionais, (ii) consumo e saúde e (iii) informação aos consumidores (Tabela 2). Quanto aos aspetos sociais,

Tabela 1 - Caracterização sociodemográfica dos participantes do estudo $(n=278)$

\begin{tabular}{|c|c|c|}
\hline Variáveis & $\mathbf{n}^{*}$ & $\%$ \\
\hline \multicolumn{3}{|l|}{ Sexo } \\
\hline Masculino & 153 & 54,9 \\
\hline Feminino & 125 & 45,1 \\
\hline \multicolumn{3}{|l|}{ Idade (anos) } \\
\hline $24-30$ & 59 & 21,5 \\
\hline $31-45$ & 83 & 30,3 \\
\hline $46-60$ & 82 & 29,9 \\
\hline Acima de 60 & 50 & 18,2 \\
\hline \multicolumn{3}{|l|}{ Nível mais alto de formação } \\
\hline Licenciatura & 128 & 46,0 \\
\hline Mestrado & 83 & 29,9 \\
\hline Doutoramento & 18 & 6,5 \\
\hline Pós-graduação & 49 & 17,6 \\
\hline \multicolumn{3}{|l|}{ Região de residência (NUTS $\|^{* *}$ ) } \\
\hline Norte & 99 & 36,3 \\
\hline Centro & 96 & 35,2 \\
\hline Lisboa & 60 & 22,0 \\
\hline Alentejo e Algarve & 2 & 4,4 \\
\hline Açores e Madeira & 6 & 2,2 \\
\hline \multicolumn{3}{|l|}{ Frequência de contacto com doentes } \\
\hline Diariamente & 243 & 87,4 \\
\hline Semanalmente & 10 & 3,6 \\
\hline Mensalmente & 1 & 0,4 \\
\hline Irregularmente & 13 & 4,7 \\
\hline Nunca, ou quase nunca & 11 & 4,0 \\
\hline \multicolumn{3}{|c|}{ Instituição de graduação universitária (licenciatura) } \\
\hline Faculdade de Medicina (Coimbra) & 93 & 33,5 \\
\hline Faculdade de Medicina (Porto) & 91 & 32,7 \\
\hline Faculdade de Medicina (Lisboa) & 28 & 10,1 \\
\hline Faculdade de Ciências Médicas (Lisboa) & 24 & 8,6 \\
\hline Outras & 39 & 14,2 \\
\hline \multicolumn{3}{|l|}{ Ano de conclusão } \\
\hline$<1982$ & 68 & 24,6 \\
\hline $1983-1993$ & 55 & 19,9 \\
\hline $1994-2004$ & 47 & 17,0 \\
\hline $2005-2016$ & 106 & 38,4 \\
\hline
\end{tabular}

*: n nem sempre totaliza o tamanho da amostra devido a inquéritos incompletos; **: Nomenclatura das Unidades Territoriais para fins estatísticos, nível 2 ambientais e nutricionais dos AT, mais de metade dos médicos concordam $(71,2 \%)$ que a engenharia genética pode ser utilizada na produção de alimentos que trazem benefícios para a humanidade, e que são uma ferramenta importante no combate à fome no mundo $(66,9 \%)$. Por outro lado, $38,5 \%$ discordam que as sementes geneticamente modificadas permitam uma agricultura mais ecológica e $49,6 \%$ discordam que as sementes geneticamente modificadas resultem numa alimentação mais saudável. Cerca de metade discorda que os AT atualmente no mercado português sejam mais nutritivos do que os seus homólogos convencionais ou biológicos $(47,4 \%)$.

Relativamente ao consumo e saúde, a maioria dos médicos $(69,4 \%)$ entende que o consumo de AT não implica problemas para a saúde, e 68,7\% acham que não é equivalente consumir alimentos transgénicos e não-transgénicos. Por outro lado, $43,8 \%$ e $49,2 \%$ dos médicos, respectivamente, concordam que o consumo de AT poderá induzir um quadro clínico ou nutricional adverso e que é provável que os alimentos transgénicos estejam associados a algum tipo de sintoma ou patologia, mesmo que tal não esteja ainda cientificamente estabelecido.

No que respeita à existência de provas científicas sobre impactos negativos para a saúde $39,9 \%$ nem concordam e nem discordam.

Em relação à questão sobre se a população está devidamente informada sobre o consumo de alimentos transgénicos, a grande maioria discorda (96\%). Uma percentagem também elevada $(60,1 \%)$ discorda que as normas atuais sejam suficientes para proteger as pessoas de eventuais perigos dos AT.

Os resultados da análise de correspondência (ANACOR; Tabela 2) entre cada uma das questões de opinião e o género, idade, nível de formação e local de residência permitiram identificar relações de dependência significativas $(p<0,05)$, criando grupos com as associações elencadas. Os principais resultados foram (i) Discordar que a engenharia genética pode ser utilizada na produção de alimentos que trazem benefícios para a humanidade (01) está associado a ser mais velho (acima dos 60 anos) e ter Mestrado; (ii) Discordar que o consumo de alimentos geneticamente modificados pode implicar problemas para a saúde (06) está associado a ser mulher e estar nas faxas etárias de 24 - 30 e 46 - 60 anos.

As opiniões dos médicos relativamente a transgénicos também foram classificadas em negativas, neutras e positivas. A maioria $(85,8 \%)$ foi classificada como tendo uma opinião neutra, $12,5 \%$ tinham uma opinião negativa e uma minoria $(1,7 \%)$ tinha uma opinião positiva.

Encontrou-se uma correlação fraca mas significativa entre estas opiniões e o nível de formação dos médicos, sendo que quanto maior o nível de formação, mais negativa é a sua opinião global em relação aos AT $\left(r_{\text {spearman }}=-0,122\right.$; $p<0,07)$. Não se encontraram correlações significativas entre a classificação da opinião e as características sociodemográficas sexo, idade e região de residência. 
Caracterização do conhecimento dos médicos em relação aos alimentos transgénicos

A avaliação do conhecimento dos médicos está sistematizada na Tabela 3. Mais de metade dos inquiridos assu- me não saber responder à maioria das questões sobre alimentos transgénicos em Portugal. A questão mais acertada $(30,7 \%)$ foi relativa à existência de rotulagem de produtos animais produzidos com recurso a rações transgénicas e a

Tabela 2 - Distribuição das respostas de opinião e resultados da análise de correspondência entre as questões e características sociodemográficas $(n=278)$

\begin{tabular}{|c|c|c|c|}
\hline \multirow[b]{2}{*}{ Questões de opinião } & \multicolumn{3}{|c|}{ Categoria da resposta } \\
\hline & $\begin{array}{l}\text { Discordo } \\
\mathrm{n}(\%)\end{array}$ & $\begin{array}{c}\text { Nem concordo/ Nem discordo } \\
n(\%)\end{array}$ & $\begin{array}{l}\text { Concordo } \\
\mathrm{n}(\%)\end{array}$ \\
\hline \multicolumn{4}{|l|}{ Aspetos sociais, ambientais e nutricionais } \\
\hline $\begin{array}{l}\text { (O1) - A engenharia genética pode ser utilizada na produção } \\
\text { de alimentos que trazem benefícios para a humanidade. }\end{array}$ & $41(14,7)^{\mathrm{d}, \mathrm{e}}$ & $39(14,0)^{\mathrm{a}, \mathrm{b}, \mathrm{g}}$ & $198(71,2)$ \\
\hline $\begin{array}{l}\text { (O2) - Os organismos geneticamente modificados são uma } \\
\text { ferramenta importante no combate à fome no mundo. }\end{array}$ & $38(13,6)$ & $47(16,9)^{\mathrm{a}}$ & $186(66,9)$ \\
\hline $\begin{array}{l}\text { (O3) - As sementes geneticamente modificadas permitem } \\
\text { uma agricultura mais ecológica. }\end{array}$ & $107(38,5)$ & $98(35,2)$ & $62(22,3)^{\mathrm{a}}$ \\
\hline $\begin{array}{l}\text { (O4) - Os alimentos transgénicos atualmente no mercado } \\
\text { português são mais nutritivos do que os seus homólogos } \\
\text { convencionais ou biológicos. }\end{array}$ & $132(47,4)$ & $114(41,0)$ & $13(4,6)^{\mathrm{a}}$ \\
\hline $\begin{array}{l}\text { (O5) - As sementes geneticamente modificadas resultam } \\
\text { numa alimentação mais saudável. }\end{array}$ & $138(49,6)^{a}$ & $103(37,0)$ & $19(6,8)$ \\
\hline \multicolumn{4}{|l|}{ Consumo e saúde } \\
\hline $\begin{array}{l}\text { (O6) - O consumo de alimentos geneticamente modificados } \\
\text { pode implicar problemas para a saúde. }\end{array}$ & $193(69,4)^{a, b, c}$ & $58(20,8)$ & $27(9,7)$ \\
\hline $\begin{array}{l}\text { (O7) - Consumir alimentos transgénicos é, na prática, } \\
\text { equivalente ao consumo de alimentos não-transgénicos. }\end{array}$ & $191(68,7)$ & $55(19,7)$ & $32(11,5)^{a}$ \\
\hline $\begin{array}{l}\text { (O8) - O consumo de alimentos geneticamente modificados } \\
\text { pode induzir um quadro clínico ou nutricional adverso. }\end{array}$ & $47(16,9)^{\dagger}$ & $109(39,2)$ & $122(43,8)$ \\
\hline $\begin{array}{l}\text { (O9) - Existem provas científicas de que os organismos } \\
\text { geneticamente modificados têm impactos negativos para a } \\
\text { saúde. }\end{array}$ & $68(24,5)^{a}$ & $111(39,9)$ & $75(27,0)$ \\
\hline $\begin{array}{l}\text { (O10) - É provável que os alimentos transgénicos estejam } \\
\text { associados a algum tipo de sintoma ou patologia, mesmo que } \\
\text { tal não esteja ainda cientificamente estabelecido. }\end{array}$ & $40(14,3)^{\mathrm{a}}$ & $83(29,8)$ & $137(49,2)$ \\
\hline \multicolumn{4}{|l|}{ Informação aos consumidores } \\
\hline $\begin{array}{l}\text { (O11) - A população está devidamente informada sobre o } \\
\text { consumo de alimentos transgénicos. }\end{array}$ & $267(96,0)$ & $5(1,8)^{f}$ & $6(2,2)$ \\
\hline $\begin{array}{l}\text { (O12) - As normas atuais são suficientes para proteger as } \\
\text { pessoas de eventuais riscos dos alimentos geneticamente } \\
\text { modificados. }\end{array}$ & $167(60,0)$ & $93(33,5)$ & $18(6,5)^{\mathrm{a}}$ \\
\hline
\end{tabular}

a-g Dependência com significância estatística (ANACOR; $p<0,05$ )

a: Feminino; b: 24 - 30 anos; c: 46 - 60 anos; d: acima de 60 anos; e: Mestrado; f: Alentejo e Algarve; g: Açores e Madeira

Tabela 3 - Distribuição das respostas de conhecimento relativamente a transgénicos em Portugal ( $n=278)$

\begin{tabular}{|c|c|c|c|c|}
\hline Questões de conhecimento & $\begin{array}{l}\text { Classificação correta } \\
\text { da afirmação }\end{array}$ & $\begin{array}{l}\text { Erraram } \\
\mathrm{n}(\%)\end{array}$ & $\begin{array}{l}\text { Não sabem } \\
\mathrm{n}(\%)\end{array}$ & $\begin{array}{l}\text { Acertaram } \\
\mathrm{n}(\%)\end{array}$ \\
\hline $\begin{array}{l}\text { (C1) - Em Portugal há rotulagem de alimentos } \\
\text { transgénicos. }\end{array}$ & Verdadeira & $55(19,9)$ & $151(54,5)$ & $71(25,6)$ \\
\hline $\begin{array}{l}\text { (C2) - Em Portugal há rotulagem de produtos } \\
\text { animais produzidos com recurso a rações } \\
\text { transgénicas. }\end{array}$ & Falsa & $26(9,4)$ & $166(59,9)$ & $85(30,7)$ \\
\hline $\begin{array}{l}\text { (C3) - Em Portugal o principal transgénico em } \\
\text { circulação é a soja. }\end{array}$ & Verdadeira & $36(13,0)$ & $163(58,8)$ & $78(28,2)$ \\
\hline $\begin{array}{l}\text { (C4) - Em Portugal o principal transgénico } \\
\text { cultivado é a soja. }\end{array}$ & Falsa & $34(12,3)$ & $184(66,4)$ & $59(21,3)$ \\
\hline $\begin{array}{l}\text { (C5) - Em Portugal vendem-se diversos } \\
\text { hortofrutícolas transgénicos. }\end{array}$ & Falsa & $107(38,6)$ & $147(53,1)$ & $23(8,3)$ \\
\hline $\begin{array}{l}\text { (C6) - Em Portugal os alimentos de agricultura } \\
\text { biológica também podem ser transgénicos. }\end{array}$ & Falsa & $150(54,2)$ & $68(24,5)$ & $59(21,3)$ \\
\hline
\end{tabular}


menos acertada $(8,3 \%)$ foi sobre a venda em Portugal de hortofrutícolas transgénicos.

O nível de conhecimento, classificado em baixo, médio e alto conforme o número de respostas acertadas ter variado entre 0 - 2, 3 - 4 e 5 - 6, respetivamente, está apresentado na Tabela 4. Os resultados mostram que $79,8 \%$ dos médicos têm um nível de conhecimento baixo, 17,7\% têm um nível de conhecimento médio e apenas $2,5 \%$ revelam um conhecimento alto.

Não se encontraram associações significativas entre cada uma das questões e o nível de conhecimento com as variáveis sociodemográficas sexo, idade, nível de formação e região de residência (ANACOR, $p>0,05$ em todos os casos).
Perceção das necessidades de formação dos médicos em relação aos alimentos transgénicos

A quase totalidade dos médicos respondentes $(92,1 \%)$ afirmou não ter recebido qualquer tipo de formação sobre AT no âmbito da sua licenciatura. No entanto, 91,4\% consideram útil existir algum tipo de preparação sobre AT durante a educação universitária e $65,1 \%$ afirma ser muito útil ao longo da vida profissional a existência de formação continuada para atualização sobre esta temática.

Os resultados da distribuição das respostas quanto às fontes de informação a que os médicos recorrem para se informarem sobre AT (Tabela 5), revistas científicas e notícias na Internet são as mais apontadas $(57,1 \%$ e $56,4 \%$, respetivamente). Contactos com especialistas e participação em congressos, palestras e seminários são as menos recorrentes $(21,5 \%$ e $24,0 \%$, respetivamente). Contudo,

Tabela 4 - Classificação do nível conhecimento dos médicos $(n=278)$

\begin{tabular}{|c|c|c|c|}
\hline \multirow[b]{2}{*}{ Característica sociodemográfica } & \multicolumn{3}{|c|}{ Nível de conhecimento } \\
\hline & $\begin{array}{c}\text { Baixo } \\
\text { n (\%) }\end{array}$ & $\begin{array}{c}\text { Médio } \\
\text { n (\%) }\end{array}$ & $\begin{array}{c}\text { Alto } \\
\mathrm{n}(\%)\end{array}$ \\
\hline \multicolumn{4}{|l|}{ Sexo } \\
\hline Masculino & $115(75,6)$ & $32(21,0)$ & $5(3,2)$ \\
\hline Feminino & $106(84,8)$ & $17(13,6)$ & $2(1,6)$ \\
\hline \multicolumn{4}{|l|}{ Idade (anos) } \\
\hline $24-30$ & $45(76,3)$ & $12(20,3)$ & $2(3,4)$ \\
\hline $31-45$ & $66(79,5)$ & $16(19,3)$ & $1(1,2)$ \\
\hline $46-60$ & $67(81,7)$ & $14(17,0)$ & $1(1,2)$ \\
\hline Acima de 60 & $40(80,0)$ & $7(14,0)$ & $3(6,0)$ \\
\hline \multicolumn{4}{|l|}{ Nível mais alto de formação } \\
\hline Licenciatura & $105(82,0)$ & $21(16,4)$ & $2(1,5)$ \\
\hline Pós-Graduação & $38(77,5)$ & $10(20,4)$ & $1(49,0)$ \\
\hline Mestrado & $65(78,3)$ & $16(19,3)$ & $2(2,4)$ \\
\hline Doutoramento & $13(76,5)$ & $2(11,76)$ & $2(11,7)$ \\
\hline \multicolumn{4}{|l|}{ Região de residência } \\
\hline Norte & $88(88,8)$ & $10(10,1)$ & $1(1,0)$ \\
\hline Centro & $74(77,0)$ & $18(18,7)$ & $4(16,6)$ \\
\hline Lisboa & $44(73,3)$ & $15(25,0)$ & $1(1,6)$ \\
\hline Alentejo e Algarve & $7(58,3)$ & $4(33,3)$ & $1(8,3)$ \\
\hline Açores e Madeira & $5(83,3)$ & $1(16,6)$ & $0(0,0)$ \\
\hline
\end{tabular}

Tabela 5 - Distribuição das respostas sobre fontes de informação a que os médicos recorrem $(n=278)$

\begin{tabular}{lccc} 
Fontes de informação sobre alimentos & \multicolumn{3}{c}{ Categoria da resposta } \\
geneticamente modificados & $\mathrm{Não}$ & $\begin{array}{c}\text { Talvez } \\
\mathrm{n}(\%)\end{array}$ & $\begin{array}{c}\text { Sim } \\
\mathrm{n}(\%)\end{array}$ \\
\hline Contacto com especialistas & $173(62,9)$ & $43(15,6)$ & $59(21,5)$ \\
Revistas científicas & $74(26,9)$ & $44(16,0)$ & $157(57,1)$ \\
Livros e documentação técnica diversa & $95(34,9)$ & $49(18,0)$ & $128(47,1)$ \\
Notícias na Internet & $66(24,0)$ & $54(19,6)$ & $155(56,4)$ \\
Comunicação social (imprensa, rádio, TV) & $107(39,1)$ & $56(20,4)$ & $111(40,5)$ \\
Congressos, palestras, seminários & $150(54,5)$ & $59(21,5)$ & $66(24,0)$ \\
Não me mantenho a par desta temática & $136(49,5)$ & $52(18,9)$ & $87(31,6)$ \\
\hline
\end{tabular}


Tabela 6 - Distribuição das respostas sobre tipologia e temas a incluir na formação académica de acordo com as preferências dos participantes. Resultados da análise de correspondência entre as questões e caracterásticas sociodemográficas $(n=278)$.

\begin{tabular}{|c|c|c|c|}
\hline \multirow[b]{2}{*}{ Questões sobre formação } & \multicolumn{3}{|c|}{ Categoria da resposta } \\
\hline & $\begin{array}{c}\text { Não } \\
\mathrm{n}(\%)\end{array}$ & $\begin{array}{c}\text { Não sei / talvez } \\
n(\%)\end{array}$ & $\underset{n(\%)}{\operatorname{Sim}}$ \\
\hline \multicolumn{4}{|l|}{ Tipologia } \\
\hline Uma disciplina opcional & $138(51)$ & $63(23,2)$ & $70(25,9)^{\mathrm{b}}$ \\
\hline Um módulo dentro de uma disciplina & $57(20,8)^{\mathrm{a}}$ & $46(16,8)$ & $171(62,3)$ \\
\hline Uma disciplina obrigatória & $190(69,1)$ & $60(21,8)$ & $25(9,1)$ \\
\hline Uma aula & $114(41,6)$ & $58(21,2)$ & $102(37,2)$ \\
\hline Um seminário curto & $60(21,8)$ & $70(25,5)$ & $145(52,7)$ \\
\hline Um trabalho teórico & $125(45,6)$ & $86(31,4)$ & $63(23,0)$ \\
\hline Um trabalho prático & $118(43,2)$ & $80(29,3)$ & $75(27,5)$ \\
\hline \multicolumn{4}{|l|}{ Temas } \\
\hline Alimentos transgénicos e genética & $12(4,4)^{\mathrm{a}}$ & $32(11,6)$ & $231(84,0)$ \\
\hline Alimentos transgénicos e rotulagem & $23(8,3)^{\mathrm{a}}$ & $62(22,5)$ & $191(69,2)$ \\
\hline Alimentos transgénicos e nutrição & $5(1,8)$ & $25(9,0)$ & $248(89,2)$ \\
\hline Alimentos transgénicos e saúde & $4(1,4)$ & $12(4,3)$ & $261(94,2)$ \\
\hline Alimentos transgénicos e ambiente & $16(5,8)$ & $64(23,2)$ & $196(71,0)$ \\
\hline Alimentos transgénicos e legislação & $26(9,4)$ & $68(24,6)$ & $182(65,9)$ \\
\hline Alimentos transgénicos e ética & $20(7,2)$ & $59(21,1)$ & $199(71,6)$ \\
\hline
\end{tabular}

a-b Dependência com significância estatística (ANACOR; $p<0,05$ )

a: Feminino; b: $24-30$ anos

$31,6 \%$ dos participantes referiram que "não se mantém a par desta temática". Não se encontraram associações significativas entre cada uma das fontes de informação e as variáveis sociodemográficas sexo, idade, nível de formação e região de residência (ANACOR, $p>0,05$ em todos os casos).

Em relação à tipologia e temas a contemplar na formação académica estão na Tabela 6. Quando questionados sobre o tipo de formação que consideravam mais adequado, a maioria $(62,3 \%)$ preferiu um módulo dentro de uma disciplina durante a licenciatura e/ou um seminário curto $(52,7 \%)$. Em relação a temas que gostariam de ver cobertos nessa formação, todas as vertentes sugeridas no inquérito foram consideradas importantes (sempre escolhidas por mais de $65 \%$ dos participantes), sendo as vertentes de saúde e de nutrição as de maior incidência $(94,2 \%$ e $89,2 \%$, respetivamente).

Os resultados da análise de correspondência (ANACOR; Tabela 6) permitiram identificar as associações seguintes entre as questões de formação e algumas características sociodemográficas ( $p<0,05)$ : (i) estar na faixa etária entre os 24 e 30 anos está associado a preferir uma disciplina opcional como tipologia de formação mais adequada; (ii) ser mulher está associado a não preferir um módulo dentro de uma disciplina e a não abordar temas de alimentos transgénicos relacionados com genética e rotulagem. Para além das questões apresentadas na Tabela 6 foi ainda incluída uma questão de resposta aberta relativa a outros temas que gostariam de ver cobertos nessa formação sobre AT. Foram apontados temas relacionados com evidências científicas, mitos, imunogenicidade, doenças autoimunes, cancro e relação com diversas fases da vida (nomeadamente em crianças e idosos).

\section{Identificação de perfis dos médicos face aos alimentos transgénicos}

Os resultados da análise de correspondência múltipla e da análise de clusters permitiram agrupar todas as questões em grupos homogéneos que possibilitaram identificar dois perfis de médicos em relação à temática dos AT. A escolha de dois perfis de respostas baseou-se numa análise de correspondência múltipla aplicada previamente, tendo sido consideradas duas dimensões que explicaram $30 \%$ da variabilidade observada nos dados. Essas duas dimensões apresentaram uma consistência interna elevada, avaliada pelo valor dos coeficientes de confiabilidade obtidos (alpha de Cronbach) que foram 0,877 e 0,835 revelando um elevado grau de homogeneidade das respostas em cada dimensão. ${ }^{13,14}$

O primeiro perfil integra $83 \%$ dos médicos. Estes médicos entendem que os AT são diferentes e não são nem mais nutritivos nem mais saudáveis quando comparados com os seus homólogos convencionais ou biológicos. Consideram que a engenharia genética não permite uma agricultura mais ecológica, que não é uma ferramenta importante no combate à fome no mundo e que não traz benefícios para a humanidade. Não têm opinião sobre se o consumo de AT pode implicar problemas para a saúde, em particular na eventual indução de um quadro clínico ou nutricional adverso. Admitem, no entanto, que estes alimentos estejam associados a algum tipo de sintoma ou patologia com impactos negativos para a saúde, embora não saibam se 
tal está cientificamente provado. Também consideram que a legislação em vigor não é suficiente para proteger a saúde. Sentem-se ignorantes quanto à realidade nacional dos AT e apoiam a possibilidade de receber formação durante a licenciatura. A sua escolha recai no formato de módulo dentro de uma disciplina. Já ao longo da vida profissional consideram a formação sobre AT muito útil, mantendo-se atualizados através da Internet e comunicação social.

O segundo perfil abrange somente $17 \%$ dos médicos. É constituído por profissionais para quem consumir AT é equivalente a consumir alimentos não-transgénicos. Consideram que a engenharia genética permite uma agricultura mais ecológica, que é uma ferramenta importante no combate à fome no mundo e que traz benefícios para a humanidade. Acreditam que o consumo de AT não acarreta qualquer tipo de problemas para a saúde e que não existem provas científicas do contrário. Quanto aos aspetos legais e nutricionais admitem não ter opinião formada. Em termos de conhecimento geral sobre AT sentem-se ignorantes e reconhecem a importância de formação na licenciatura, elegendo uma aula ou um seminário curto como formatos preferíveis. Não consideram relevante formação ao longo da vida.

\section{DISCUSSÃO}

Este é o primeiro estudo a detalhar a opinião, o conhecimento e necessidades de formação relativamente aos AT. As hipóteses de que (i) os médicos possuem conhecimento insuficiente sobre os alimentos transgénicos, que (ii) há pontos de vistas opostos e (iii) há necessidade de formação, foram confirmadas para a população inquirida.

\section{Opinião}

Em relação à opinião dos médicos portugueses foi possível perceber que a maioria tem uma posição neutra sobre AT. Opiniões positivas foram encontradas no estudo de Schmidt et $a l,{ }^{16}$ realizado com 241 profissionais de saúde (sendo 43 médicos) nos Estados Unidos da América, baseado num inquérito sobre atitudes, crenças e preocupações face à biotecnologia e AT. Globalmente os resultados indicaram que uma maioria dos respondentes concordaram que a biotecnologia alimentar tem o potencial de afetar positivamente a saúde humana nos próximos 20 anos (55\%) e que alimentos funcionais geneticamente modificados podem contribuir para reduzir riscos de doença (51\%).

Resultados diferentes e relativos à opinião de profissionais de saúde foram encontrados na Turquia. Burcu, ${ }^{17}$ num inquérito realizado a 307 profissionais de saúde em formação na área da obstetrícia, concluiu que a maioria dos indivíduos revelou ter opiniões negativas sobre AT. Savas et $a l,{ }^{18}$ num estudo sobre AT que envolveu 200 médicos turcos, verificou que $80,5 \%$ dos médicos inquiridos definiram os AT como prejudiciais. Resultados semelhantes foram publicados por Koçak et al, ${ }^{19}$ onde $56,9 \%$ dos 427 estudantes de medicina participantes consideraram que a produção de AT é arriscada para todos os organismos vivos na natureza. No estudo de Ergin et $a l,{ }^{20}$ envolvendo também estudantes de medicina, cerca de $76 \%$ dos respondentes consideraram que AT podem prejudicar a saúde humana e $79 \%$ acharam que não podem ser consumidos com segurança.

As diferentes opiniões encontradas na classe médica podem advir do desconhecimento das evidências científicas sobre AT ou do que elas efetivamente demonstram, além da inexperiência de se posicionarem. Atualmente os AT em circulação no mercado português estão autorizados para consumo humano. ${ }^{9}$ Há já estudos que apontam para impactos negativos na saúde. ${ }^{21,22}$ Outras preocupações estão relacionadas com questões ambientais ${ }^{23,24}$ e éticas, ${ }^{25,26}$ o que possivelmente leva grupos específicos a duvidar dos atuais resultados científicos onde se conclui que os AT são seguros. ${ }^{21,22} \mathrm{Na}$ questão ambiental possivelmente o uso de sementes resistentes a pragas pode levar as ervas daninhas e herbívoros a adquirirem resistência. Essa resistência pode acarretar um maior uso de agrotóxicos e, consequentemente, aumenta os resíduos desses produtos nos alimentos e no próprio ambiente, desencadeando desequilíbrios ecológicos. ${ }^{23,24}$ Já dentro das questões éticas leva em conta, alguns princípios em debate, como, por exemplo do princípio da justiça (irreversibilidade face aos mais desfavorecidos, face ainda aos que não nasceram), princípio da não maleficência (garantia de segurança versus possibilidade de segurança) e princípio do respeito (direito a saber o que se semeia e come). ${ }^{25,26}$

\section{Conhecimento}

O conhecimento dos médicos portugueses face aos AT, de acordo com a classificação utilizada neste estudo, pode classificar-se como baixo. Resultados semelhantes foram apresentados por Savas et $a l,{ }^{18}$ onde $40 \%$ dos médicos respondentes consideraram o seu nível de conhecimento sobre AT como sendo mau ou muito mau. Ergin et al, ${ }^{20}$ relativamente a 377 estudantes de medicina, verificaram que $81,4 \%$ dos indivíduos inquiridos avaliou o seu conhecimento sobre AT como sendo insuficiente. Keskin et a ${ }^{27}$ concluíram que os estudantes de medicina apresentam um nível de conhecimento baixo sobre os efeitos de AT nos seres humanos e que devem ter formação. Já Burcu, ${ }^{17}$ relativamente aos profissionais de saúde em formação, concluiu que o seu conhecimento sobre AT era satisfatório.

Os profissionais de saúde em geral, e os médicos em particular, são considerados fontes de informação fiável (Wunderlich e Gatto ${ }^{28}$ a quem a população opta por recorrer para obter informação sólida sobre alimentos e saúde, o que acresce à responsabilidade de garantir um conhecimento suficiente sobre AT.

\section{Formação}

Os resultados deste estudo parecem fundamentar e justificar a inclusão de um módulo sobre AT dentro de uma unidade curricular na licenciatura de medicina. Subtemas relacionados com genética, nutrição, saúde, ambiente, rotulagem, legislação e ética foram apontados como de interesse por parte da grande maioria dos médicos 
inquiridos. Estes conteúdos permitirão preencher as lacunas de informação sobre os AT, proporcionando uma visão holística desde a sua produção até à mesa do consumidor. Uma abordagem multidisciplinar permitirá, também, uma conexão de conceitos técnicos de engenharia genética com as dimensões que transcendem a ciência no sentido estrito, como a aceitação do risco, o direito à informação e as opções legadas às gerações vindouras, entre outros. Estudos de Schmidt et $a l,{ }^{16}$ Ergin et $a^{20}$ e Koçak et $a^{19}$ corroboram as necessidades de formação dos médicos, identificadas neste trabalho.

Os meios mencionados como fontes de informação a que os médicos portugueses recorrem para se manterem atualizados sobre AT contribuem para uma melhor gestão das estratégias de comunicação e formação. Revistas científicas e Internet foram os mais apontados, tal como em Savas et $a l,{ }^{18}$ onde a Internet é referida como fonte de informação preferencial por $23 \%$ dos médicos inquiridos. Revistas científicas e programas de educação continuada são indicados por $71 \%$ dos respondentes no estudo de Schmidt et al, ${ }^{16}$ Já as redes sociais são os mencionados por $72,5 \%$ dos inquiridos por Burcu, ${ }^{17}$ enquanto Koçak et al ${ }^{18}$ constataram que $67,8 \%$ obtêm informações por meio da televisão e rádio.

\section{Perfis dos médicos face aos alimentos transgénicos}

Este estudo demonstrou que os médicos se dividem em dois grupos face aos AT, distinguindo-se na opinião, confiança e interesse relativamente a esta temática. Um dos grupos, que abrange a grande maioria dos médicos (83\%), apresenta um perfil interessado em saber mais sobre AT, assumindo à partida uma posição desfavorável e de desconfiança. Outro grupo, minoritário (17\%), integra um perfil desinteressado, confiante e favorável aos AT. A caracterização destes perfis ajuda a perceber o posicionamento da classe médica face a esta temática e pode orientar futuras estratégias de formação.

Os AT representam um saber científico atualmente em crescimento, onde os traços de identidade disciplinar, academica e profissional estão sendo ainda formatados. Eles convergem para o reconhecimento de uma área de trabaIho importante, visto o impacto direto na saúde e no meio ambiente (seja positivo e/ou negativo). Este trabalho contribui para a literatura atual sobre posicionamento dos profissionais de saúde relativamente à temática dos AT. Os resultados deste estudo efetivamente avançam outras investigações desenvolvidas até à data. Do que foi exposto seria interessante desenvolver outros estudos de investigação, no domínio de ensino-aprendizagem em alimentos transgénicos para profissionais de saúde, na medida em que esse tipo de investigação é escasso.

O presente trabalho empregou uma amostra de conveniência, pelo que é necessário cautela em qualquer gene- ralização. No entanto, os resultados preenchem uma lacuna na literatura atual sobre a relação dos médicos com os AT. Sendo claras as implicações dos resultados apresentados para qualquer revisão futura do currículo académico em faculdades de medicina, as consequências últimas de tal processo serão sentidas pelos pacientes, em resultado de uma melhoria da competência e prática clínica nesta vertente. Espera-se que investigação futura permita clarificar a melhor estratégia para garantir que os profissionais de saúde em geral e os médicos em particular aprendem a lidar com a rapidez de evolução, incerteza intrínseca e sobretudo ignorância das consequências - impossíveis de antecipar - que uma tecnologia tão recente e tão potente como a engenharia genética efetivamente acarreta.

\section{CONCLUSÃO}

Este estudo é pioneiro no que se refere ao posicionamento de médicos portugueses relativamente aos AT. Os resultados evidenciaram a polarização entre um grupo claramente maioritário (acima dos $80 \%$ ) que vê os AT com ceticismo e está interessado em conhecer mais sobre o assunto, e uma minoria (inferior a $20 \%$ ) que confia na tecnologia ao ponto de ser favorável à sua introdução sem se sentir impelida a procurar informação adicional.

$\mathrm{Na}$ generalidade, os resultados evidenciaram um baixo nível de conhecimento dos médicos face aos AT o que aponta para uma necessidade de formação nesta temática, eventualmente a colmatar sob a forma de inclusão de um módulo especializado no âmbito de uma unidade curricular preexistente na licenciatura. Subtemas relacionados com genética, nutrição, saúde, ambiente, rotulagem, legislação e ética foram apontados como de interesse para a formação médica.

\section{PROTEÇÃO DE PESSOAS E ANIMAIS}

Os autores declaram que os procedimentos seguidos estavam de acordo com os regulamentos estabelecidos pelos responsáveis da Comissão de Investigação Clínica e Ética e de acordo com a Declaração de Helsínquia da Associação Médica Mundial.

\section{CONFIDENCIALIDADE DOS DADOS}

Os autores declaram ter seguido os protocolos do seu centro de trabalho acerca da publicação de dados.

\section{CONFLITO DE INTERESSES}

Os autores declaram não ter qualquer conflito de interesse relativamente ao presente artigo.

\section{FONTES DE FINANCIAMENTO}

Os autores declaram não ter recebido qualquer subsídio ou bolsa relativamente ao presente artigo.

\section{REFERÊNCIAS}

1. Zhang $\mathrm{C}$, Wohlhueter R, Zhang H. Genetically modified foods: a critical review of their promise and problems. Food Sci Hum Well. 2016;5:116- 
allergenicity of genetically modified foods from genetically engineered crops. Ann Allergy Asthma Immunol. 2017;119:214-22.

3. Comissão Europeia. Directiva 2001/18/CE do parlamento europeu e do conselho de 12 de março de 2001 relativa à libertação deliberada no ambiente de organismos geneticamente modificados e que revoga a directiva 90/220/CEE do conselho. Bruxelas: CE; 2001.

4. Lucht JM. Public acceptance of plant biotechnology and GM crops. Viruses. 2015;7:4254-81.

5. Frewer LJ, Van der Lansb IA, Fischerb AR, Reindersc MJ, Menozzid D, Zhangc $X$, et al. Public perceptions of agri-food applications of genetic modification - a systematic review and meta-analysis. Trends Food Sci Technol. 2013;30:142-52.

6. International Service for the Acquisition of Agri-biotech Applications. Global Status of Commercialized Biotech/GM Crops in 2017: Biotech Crop Adoption Surges as Economic Bene ts Accumulate in 22 Years. Ithaca: ISAA; 2017

7. Ec.europa.eu (homepage na internet). Portugal: EU Register of Authorised GMOs. 2017; [consultado 2018 jul 30]. Disponível em: http:// ec.europa.eu/food/.

8. World Health Organization. Modern food biotechnology, human health and development : an evidence-based study. Geneva: WHO; 2005.

9. Uzogara SG. The impact of genetic modification of human foods in the 21st century: a review. Biotechn Adva. 2000;18:179-206.

10. Paparini A, Romano-Spica V. Public health issues related with the consumption of food obtained from genetically modified organisms. Biotechnol Annu Rev. 2004;10:85-122.

11. Key S, Ma KC, Drake PM. Genetically modified plants and human health. J R Soc Med. 2008;101:290-8.

12. Sanchez MA, Parrott WA. Characterization of scientific studies usually cited as evidence of adverse effects of GM food/feed. Plant Biotechnol J. 2017;15:1227-34.

13. Pestana $\mathrm{MH}$, Gageiro JN. Análise de dados para ciência sociais - a complementariedade do SPSS. $6^{a}$ ed. Lisboa: Editora Sílabo; 2014.

14. Carvalho $\mathrm{H}$. Análise multivariada de dados qualitativos - utilização da ACM com o SPSS. $1^{\mathrm{a}}$ ed. Lisboa: Editora Manuel Robalo; 2008.

15. Conchran WG. Técnicas de amostragem (tradução). Rio de Janeiro: Fundo de Cultura; 1965.
16. Schmidt JL, Vickery CE, Cotugna NA, Snider OS. Health professionals hold positive attitudes toward biotechnology and genetically engineered foods. J Environ Health. 2005;67:44-9.

17. Burcu YZ. Midwifery students' knowledge and opinions about and behaviors towards biotechnology. Int J Caring Sci. 2017;10:963-70.

18. Savas HB, Gultehin F, Doguc DK, Oren O, Guler M, Demiralay H. Medical doctors ' perceptions os genetically modified foods. J Clin Analyt Med. 2014;7:1-4

19. Koçak N, Turker T, Kilic S, Hasde M. Assessment of knowledge, attitude and behavior level of medical school students about genetically modified organisms. Gulhane Med J. 2010;52:198-204.

20. Ergin A, Uzun SU, Bozkurt AL. Pamukkale university faculty of medicine students' knowledge and opinions regarding genetically modified organisms. Pamukkale Med J. 2015;8:92-8.

21. Hug K. Genetically modified organisms: do the benefits outweigh the risks? Medicina. 2008;44:87-99.

22. Ozkok GA. Genetically modified foods and the probable risks on human health. Int J Nutr Food Sci. 2015;4:356-63.

23. Tsatsakis AM, Nawaz MA, Tutelyan VA, Golokhvast KS, Kalantzi OL, Chung $\mathrm{DH}$, et al. Impact on environment, ecosystem, diversity and health from culturing and using GMOs as feed and food. Food Chem Toxicol. 2017;107:108-21

24. Kramkowska M, Grzelak T, Czyżewska K. Benefits and risks associated with genetically modified food products. Ann Agric Environ Med. 2013;20:413-9.

25. Dizon F, Costa S, Rock C, Harris A, Husk C, Mei J. Genetically modified (GM) foods and ethical eating. J Food Sci. 2016.81:1287-91.

26. Glass S, Fanzo J. Genetic modification technology for nutrition and improving diets: an ethical perspective. Curr Opin Biotech. 2017;44:4651.

27. Keskin $\mathrm{Y}$, Luleci N, Ozyaral O, Altinta O, Saglik A, Lisar H, et al. Knowledge attitude and behavior of the students in medical school of maltepe university about genetically modified organisms. Maltepe Med J. 2010;2:14-23.

28. Wunderlich S, Gatto Kelsey A. Consumer perception of genetically modified organisms and sources of information. Adv Nut. 2015;6:84251. 